\title{
Introduction to the Special Issue on Multimodal Machine Learning for Human Behavior Analysis
}

Analyzing human behaviors in multimedia data has become one of the most interesting topics in intelligent multimedia perception. Recently, with the widespread availability of advanced visual and nonvisual sensors and a growing need for a user-friendly interface, integrating multimodality data for human behavior analysis, has received a great deal of research interests from the community of multimedia analysis. Compared to the traditional single-modality human behavior analysis, multimodality human behavior analysis provides deeper-level understanding of human identification and event detection, and a more comprehensive perspective for understanding the intrinsic interaction and connections of humans.

Although the studies of human behavior analysis in multimodality data are invaluable for both academia and industry, there are many fundamental problems unsolved so far, such as learning representation of human appearance and behaviors from multiple modalities, mapping data from one modality to another to achieve cross-modality human behavior analysis, identifying and utilizing relations between elements from two or more different modalities for comprehensive behavior analysis, fusing information from two or more modalities to perform a more accurate prediction, transferring knowledge between modalities and their representations, and recovering missing modality data given the observed ones.

This special issue accepted nine articles that address the challenging issues of multimodal machine learning for human behavior analysis. The first three articles focus on modeling multiple features using sparse coding. In particular, "Robust Visual Tracking Using Kernel Sparse Coding on Multiple Covariance Descriptors" proposes to use covariance matrices as descriptors to represent multiple features and then performs tracking in a sparse representation framework. "CovLets: A Second Order Descriptor for Modeling Multiple Features" proposes to aggregate local descriptors in the form of covariance descriptors into a rich descriptor by using sparse coding to learn second-order statistics of the covariance descriptors. "Action Recognition Using Form and Motion Modalities" uses hierarchal sparse coding to learn the underlying features from videos. The learned features characterize the form and motion simultaneously and therefore provide more accurate and complete feature representation.

Beside sparse coding, six deep learning-based methods have also been proposed to address some issues of multimodal feature learning. "AMIL: Adversarial Multi-Instance Learning for Human Pose Estimation" proposes generative adversarial networks as the learning model, which has two residual multiple instance learning models with identical architecture. One is used as the generator, and the other is used as the discriminator. In addition to generative adversarial networks, "Multichannel Attention Refinement for Video Question Answering" proposes to tackle the Video Question Answering task-that is, the extension of image question answering in the video-from

\footnotetext{
ACM Reference format:

https://doi.org/10.1145/3381917

(C) 2020 Copyright held by the owner/author(s).

1551-6857/2020/03-ART19

https://doi.org/10.1145/3381917
}

Shengping Zhang, Huiyu Zhou, Dong Xu, M. Emre Celebi, and Thierry Bouwmans. 2020. Introduction to the Special Issue on Multimodal Machine Learning for Human Behavior Analysis. ACM Trans. Multimedia Comput. Commun. Appl. 16, 1s, Article 19 (March 2020), 2 pages. 
a multichannel perspective. Appearance, motion, and audio features are extracted from the video, and question-guided attentions are refined to generate the expressive clues that support the correct answer. "Delving Deeper in Drone-Based Person Re-Id by Employing Deep Decision Forest and Attributes Fusion" uses a deep neural decision forest for pedestrian classification, which employs the $\mathrm{CBOW}$ model to extract the word embeddings from text descriptions and fuse it with features extracted from images. In "Spatial Preserved Graph Convolution Networks for Person ReIdentification," a graph convolution network-based model for person re-identification is proposed to learn more discriminative feature embeddings, where a graph-structured relationship between person images and person parts are integrated. "ACMNet: Adaptive Confidence Matching Network for Human Behavior Analysis via Cross-Modal Retrieval” proposes an adaptive confidence matching network that introduces a confidence score for the local similarity by leveraging the global text(/image) information. The proposed method is expected to help measure the semantic relatedness of the region(/word) to the whole text(/image). "Multi-Scale Supervised Attentive Encoder-Decoder Network for Crowd Counting” proposes a novel attentive encoderdecoder network for crowd counting. The attentive encoder-decoder network is better at establishing long-range dependencies between the encoder and decoder, therefore promoting more effective fusion of multiscale features for handling scale variations.

Shengping Zhang
Harbin Institute of Technology, China
Huiyu Zhou
School of Informatics, University of Leicester, United Kingdom
Dong Xu
University of Sydney, Australia
M. Emre Celebi
University of Central Arkansas, United States
Thierry Bouwmans
University of La Rochelle, France
Guest Editors

